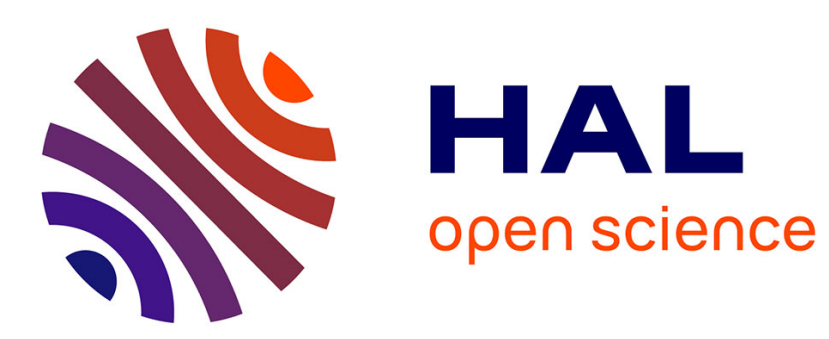

\title{
Is a Brownian motion skew?
}

Antoine Lejay, Ernesto Mordecki, Soledad Torres

\section{To cite this version:}

Antoine Lejay, Ernesto Mordecki, Soledad Torres. Is a Brownian motion skew?. Scandinavian Journal of Statistics, 2014, 5 (2), pp.346-364. 10.1111/sjos.12033 . inria-00544442v4

\section{HAL Id: inria-00544442 https://hal.inria.fr/inria-00544442v4}

Submitted on 19 Jul 2013

HAL is a multi-disciplinary open access archive for the deposit and dissemination of scientific research documents, whether they are published or not. The documents may come from teaching and research institutions in France or abroad, or from public or private research centers.
L'archive ouverte pluridisciplinaire HAL, est destinée au dépôt et à la diffusion de documents scientifiques de niveau recherche, publiés ou non, émanant des établissements d'enseignement et de recherche français ou étrangers, des laboratoires publics ou privés. 


\section{Is a Brownian motion skew?}

ANTOINE LEJAY ${ }^{1}$

Université de Lorraine, IECN, UMR 7502, Vandœuvre-lès-Nancy, F-54500, France

CNRS, IECL, UMR 7502, Vandœuvre-lès-Nancy, F-54500, France

Inria, Villers-lès-Nancy, F-54600, France

ERNESTO MORDECKI ${ }^{2}$

Centro de Matemática, Facultad de Ciencias, Universidad de la República

SOLEDAD TORRES ${ }^{3}$

CIMFAV, Facultad de Ingeniería, Universidad de Valparaíso

May 10, 2013

Abstract: We study the asymptotic behavior of the maximum likelihood estimator corresponding to the observation of a trajectory of a Skew Brownian motion, through a uniform time discretization. We characterize the speed of convergence and the limiting distribution when the step size goes to zero, which in this case are non-classical, under the null hypothesis of the Skew Brownian motion being an usual Brownian motion. This allows to design a test on the skewness parameter. We show that numerical simulations can be easily performed to estimate the skewness parameter, and provide an application in Biology.

Keywords: maximum likelihood, skew Brownian motion, statistical estimation,

\section{Introduction}

The Skew Brownian Motion (SBm) has attracted interest within other facts, due to its relations with diffusions with discontinuous coefficients or to media with permeable barriers, being the first example of the solution of a stochastic differential equation with the local time of the solution as drift (Harrison \& Shepp, 1981): the SBm $X=\left\{X_{t}: 0 \leq t \leq T\right\}$ can be defined as the strong solution of the stochastic differential equation

$$
X_{t}=x+B_{t}+\theta \ell_{t}
$$

where $B=\left\{B_{t}: 0 \leq t \leq T\right\}$ is a standard Brownian motion defined on a probability space $(\Omega, \mathcal{F}, \mathbb{P})$, the initial condition is $x \geq 0$ (the case $x<0$ is symmetrical), $\theta \in[-1,1]$ is the skewness parameter, and $\ell=\left\{\ell_{t}: 0 \leq t \leq T\right\}$ is the local time at level zero of the (unknown) solution $X$ of the equation departing from $x$, defined by

$$
\ell_{t}=\lim _{\epsilon \rightarrow 0} \frac{1}{2 \epsilon} \int_{0}^{t} \mathbf{1}_{(-\epsilon, \epsilon)}\left(X_{s}\right) d s .
$$

In the literature the skewness parameter is sometimes defined as $p=(\theta+1) / 2$; this second parametrization being more convenient for an alternative construction of the SBm:

\footnotetext{
${ }^{1}$ This author has been supported by the MathAmSud program and the Inria Équipe Associée AnestocTosca.

${ }^{2}$ The author's research is supported by CMAT-UDELAR, and by project SAMP Network, of the MathAmSud program.

${ }^{3}$ The author was supported by Dipuv grant 26/2009, Fondecyt Grant N. 1130586, ACT1112, CONICYT-PIA: Stochastic Analysis Research Network.
} 
depart from the reflected Brownian motion and choose, independently with probability $p \in[0,1]$, whether each particular excursion of the reflected Brownian motion remains positive.

In the special case $\theta=1(p=1)$, the solution to (1) is the reflected Brownian motion. The case $\theta=0(p=1 / 2)$ corresponds to the the standard Brownian motion.

Recently, several papers have considered the SBm in modelling or simulation issues, as well as some optimization problems. Thanks to the Itô-Tanaka formula, the SBm is strongly related to diffusion processes whose infinitesimal generator, both divergenceform or non divergence-form, has discontinuous coefficients (Lejay \& Martinez, 2006). Hence, the SBm appears naturally for example in geophysics as a model of the diffusion of a pollutant (Ramirez et al., 2006), in brain imaging by diffusion MRI or in electro/encephalography (Martinez, 2004), in population ecology (Cantrell \& Cosner, 1999; Ovaskainen \& Cornell, 2003), in astrophysics (Zhang, 2000). See the review by Lejay (2006) for references on the subject, as well as a survey of the various possible constructions and applications of the SBm. In Lejay \& Pichot (2012), it is shown how to simulate exactly the position of the SBm after a constant time step.

In this paper we are interested in the statistical estimation of $\theta$, the skewness parameter, when we observe a trajectory of the process through an equally spaced time grid. From the statistical point of view we find this problem interesting because it is in certain sense intermediate between the classical problem of drift estimation in a diffusion, where the measures generated by the trajectories of the process for different values of the parameter are equivalent (Kutoyants, 1984; Lipster \& Shiryaev, 2001), and the estimation of the variance (the volatility in financial terms) of a diffusion (see for instance Florens-Zmirou (1993), or Jacod (2006) and the references therein), where the probability measures generated by the trajectories are singular for different values of the parameter. At the best of our knowledge, the only estimator of $\theta$ is the one constructed in Bardou \& Martinez (2010), where the authors assume that the SBm is reflected at levels 1 and -1 to ensure ergodicity, considering a different scheme of observation of the trajectory.

The strategy proposed by O. Bardou and M. Martinez does not apply here, as it is strongly related to ergodicity while the SBm is only null recurrent. The distribution of the renormalized occupation time on the positive side $A^{+}(t) / t$ of the SBm up to time $t$ is computed in Lamperti (1958) (for $\theta=0$, this is the famous Arc-Sine distribution). Despite the mean of $A^{+}(t) / t$ is equal to $(1+\theta) / 2$, it has a positive variance and it may not be used to set up an estimator of $\theta$, nor its discretized version (See Fujihara et al. (2007) for example).

Indeed, only the behavior of the SBm close to 0 brings some information for estimating $\theta$. The expansion the log-likelihood provides indeed some weighted version of the difference between upcrossings and downcrossings.

The main results of this article follows. First, we prove that under the distribution of the Brownian motion, that is with $\theta=0$, the MLE estimator $\theta_{n}$ from $n$ equally spaced observations of $X_{k T / n}$ on $[0, T]$ converges in probability to the true value $\theta=0$. Second, we prove that the scaled estimator $n^{1 / 4} \theta_{n}$ converges stably to $c T^{1 / 4} W\left(\ell_{T}\right) / \ell_{T}$ when the trajectory hits zero, where $c$ is a constant and $W$ is a Brownian motion independent from $X$. The convergence of $n^{1 / 4} \theta_{n}$ is the Local Asymptotically Mixed Normality (LAMN) property at the point $\theta=0$ (Le Cam \& Yang, 2000), but with a rate $1 / 4$ while in general, the rate is $1 / 2$. This situation is typical for null recurrent estimation problems (Höpfner \& Löcherbach, 2003). Third, we obtain an expansion of $\theta_{n}$ as $\theta_{n}^{(1)} / n^{1 / 4}+\theta_{n}^{(2)} / n^{1 / 2}+$ $\theta_{n}^{(3)} / n^{3 / 4}+\cdots$ where the $\theta_{n}^{(i)}$ are computed recursively. 
Numerical simulations show us that it is plausible that $\theta_{n}$ converges in probability to $\theta$ under the distribution of the SBm of parameter $\theta$. However, we have not been able to prove that $\theta^{n}$ converges to $\theta$ in probability in such a situation. The key argument we used for $\theta=0$, which are contained in some limit theorem studied in Jacod (1998), are difficult to extend here, as the distribution of the SBm for $\theta=0$ is singular with respect to the one of the Brownian motion (Le Gall, 1984). However, if all the observations are positive (resp. negative), then the MLE estimator gives 1 (resp. -1). Hence, under the distribution of the reflected Brownian motion, the estimator always gives the right value of $\theta$, but this corresponds to a degenerate case.

The rest of the paper is organized as follows. Section 2 describes the maximum likelihood methodology and the convergence results. In Section 3 we describe the limit distribution. Section 4 presents a statistical test and some numerical simulations on the likelihood function. Section 5 presents an application to diffusion of species in two different habitats, and Section 6 our conclusions. Finally, in the Appendix we provide the theorems from Jacod (1998) used in the proof of our main results in Section 2.

\section{The maximum likelihood estimator}

Consider the $\operatorname{SBm} X$ with parameter $\theta \in[-1,1]$ defined in (1) and the sampling scheme denoted by $X_{i}:=X_{i T / n}(i=0, \ldots, n)$, and $\Delta=T / n$. In this section we derive the asymptotic behaviour of maximum likelihood estimator $\theta_{n}$ of the parameter $\theta$ when we observe the sample $X_{1}, \ldots, X_{n}$. As shown in Walsh (1978), the transition density of the SBm of parameter $\theta \in[-1,1]$ is given by:

$$
q_{\theta}(t, x, y):=p(t, y-x)+\operatorname{sgn}(y) \theta p(t,|x|+|y|),
$$

where

$$
p(t, x)=\frac{1}{\sqrt{2 \pi t}} \exp \left(-\frac{x^{2}}{2 t}\right)
$$

is the density of a Gaussian random variable with variance $t$ and mean 0 and $\operatorname{sgn}(x)=1$ if $x>0, \operatorname{sgn}(x)=-1$ if $x<0$ and $\operatorname{sgn}(x)=0$ if $x=0$. The likelihood of the sample is given by

$$
\Lambda_{n}(\theta):=\prod_{i=0}^{n-1} q_{\theta}\left(\Delta, X_{i}, X_{i+1}\right) .
$$

The maximum likelihood estimator (MLE) is

$$
\theta_{n}:=\operatorname{argmax}_{\theta \in[-1,1]} \Lambda_{n}(\theta)
$$

The uniqueness of the MLE follows from simple computations.

Lemma 1. For each $n$, there exists a unique $M L E \theta_{n}$.

Proof. The derivatives of $L_{n}(\theta)=\log \left(\Lambda_{n}(\theta)\right)$ with respect to $\theta$ are easily computed thanks to the simple expression of the density $q_{\theta}$ in $(2)$. As seen in $(3), \partial_{\theta}^{2} L_{n}(\theta)<0$. This proves that $\theta \mapsto L_{n}(\theta)$ is strictly concave so that $\theta_{n}$ is unique.

Remark 1. The SBm has the same scaling property as the Brownian motion. If $X$ is a SBm of parameter $\theta$, so is $c X_{t / c^{2}}$ for $c>0$. Hence, for fixed $n$ and $T, Y=\left\{\sqrt{n / T} X_{t T / n}: t \in\right.$ $[0, n]\}$ with $X_{0}=0$ is a $\mathrm{SBm}$ of parameter $\theta$ for a $\mathrm{SBm} X$ of parameter $\theta$. It is then 
easily seen that the maximum likelihood estimator applied to the sequence $\left\{Y_{i}\right\}_{i=0, \ldots, n}$ with a time-step $\Delta=1$ is distributed as the one for the sequence $\left\{X_{i T / n}\right\}_{i=0, \ldots, n}$ with a time-step $\Delta=T / n$. In this case, high-frequency estimation and long-time estimation are equivalent. In any case, we are in the situation of a statistical estimation problem for a null-recurrent Markov process.

The difference between a SBm and a Brownian motion lies at $x=0$, the origin, so that the path should cross 0 in order to get a meaningful estimator.

Lemma 2. If all the $X_{i}$ 's are positive (resp. negative) for $i=1, \ldots, n$, then $\theta_{n}=1$ (resp. $\left.\theta_{n}=-1\right)$. This means that the estimator sees a positively (resp. negatively) reflected Brownian motion.

Proof. From (2), for fixed $x \in \mathbb{R}$ and $y \neq 0, \operatorname{argmax} q_{\theta}(\Delta, x, y)=\operatorname{sgn}(y)$. Besides, if $y=0$, then $p_{\theta}(\Delta, x, 0)$ does not depend on $\theta$. Hence, if all the observations $X_{1}, \ldots, X_{n}$ are non-negative (resp. non-positive), then $\theta_{n}=1$ (resp. $\theta_{n}=-1$ ) since $\Lambda_{n}(\theta)$ is the product of the $q_{\theta}\left(\Delta, X_{i}, X_{i+1}\right)$ 's. We exclude the degenerated case that all the $X_{i}$ 's are equal to 0 , which is almost surely never observed whatever the value of $\theta$.

Lemma 3. If $\theta_{n}$ is the MLE associated to $\left\{X_{i}\right\}_{i=0, \ldots, n}$, then $-\theta_{n}$ is the MLE associated to $\left\{-X_{i}\right\}_{i=0, \ldots, n}$.

Proof. This is an easy consequence of (2): The likelihood is left unchanged when the signs of $\theta$ and of the $X_{i}$ 's are changed.

\subsection{The log-likelihood around $\theta=0$}

Instead of using the likelihood $\Lambda_{n}(\theta)$, it is often more convenient to use the log-likelihood defined by

$$
L_{n}(\theta):=\log \Lambda_{n}(\theta)=\sum_{i=0}^{n-1} \log q_{\theta}\left(\Delta, X_{i}, X_{i+1}\right) .
$$

The MLE is the point $\theta_{n}$ at which $\theta \mapsto L_{n}(\theta)$ reaches its maximum. The MLE $\theta_{n}$ may equivalently be defined as the solution to $L_{n}^{(1)}\left(\theta_{n}\right)=0$.

For $k \geq 1$, its scaled (for notational convenience) $k$-th derivatives are

$$
L_{n}^{(k)}(\theta)=\frac{1}{(k-1) !} \frac{\partial^{k}}{\partial \theta^{k}} L_{n}(\theta)
$$

that are computed as

$$
L_{n}^{(k)}(\theta)=(-1)^{k-1} \sum_{i=0}^{n-1} \frac{\operatorname{sgn}\left(X_{i+1}\right)^{k} p\left(\Delta,\left|X_{i}\right|+\left|X_{i+1}\right|\right)^{k}}{q_{\theta}\left(\Delta, X_{i}, X_{i+1}\right)^{k}} .
$$

An analytic development of $L_{n}^{(1)}(\theta)$ holds around 0 :

$$
L_{n}^{(1)}(\theta)=\sum_{k=0}^{+\infty} \theta^{k} L_{n}^{(k+1)}(0)
$$

The inequality

$$
\frac{p(\Delta,|x|+|y|)}{q_{0}(\Delta, x, y)}=\exp \left(-\frac{|x y|+x y}{\Delta}\right)=\exp \left(\frac{-2(x y)^{+}}{\Delta}\right) \leq 1, x, y \in \mathbb{R}
$$


implies that $\left|L_{n}^{(k)}(0)\right| \leq n$ and thus the series in (4) is absolutely convergent for $|\theta|<1$.

Introduce, for $k=1,2, \ldots$, the sequence of functions

$$
h_{k}(x, y)=h(x, y)^{k} \text { with } h(x, y)=\operatorname{sgn}(x+y) \exp \left(-(2 / T)(x(x+y))^{+}\right) .
$$

We can then rewrite $L_{n}^{(k)}(0)$, for $k=1,2, \ldots$, as:

$$
L_{n}^{(k)}(0)=(-1)^{k-1} \sum_{i=0}^{n-1} h_{k}\left(\sqrt{n} X_{i}, \sqrt{n}\left(X_{i+1}-X_{i}\right)\right) .
$$

We then see that the study of the limit behaviour of this type of sums, presented in the next proposition, can be directly obtained from results in Jacod (1998) (For convenience, we present these results in an Appendix).

\subsection{Asymptotic behavior of the MLE under the Brownian distri- bution}

Let us set

$$
\alpha_{n}:=-n^{1 / 4} \frac{L_{n}^{(1)}(0)}{L_{n}^{(2)}(0)}=-n^{1 / 4} \frac{\sum_{i=0}^{n-1} \operatorname{sgn}\left(X_{i+1}\right) h\left(\sqrt{n} X_{i}, \sqrt{n}\left(X_{i+1}-X_{i}\right)\right)}{\sum_{i=0}^{n-1} h\left(\sqrt{n} X_{i}, \sqrt{n}\left(X_{i+1}-X_{i}\right)\right)^{2}} .
$$

Unlike the MLE, $\alpha_{n}$ is pretty simple to compute from the $X_{i}$ 's.

For a non-negative starting point $x=X_{0}$, we consider the events

$$
\begin{aligned}
A_{n} & :=\left\{\omega: \inf _{1 \leq i \leq n} X_{i}(\omega)<0 \text { and } \sup _{1 \leq i \leq n} X_{i}(\omega)>0\right\} \\
A & :=\left\{\omega: \inf _{0 \leq t \leq T} X_{t}(\omega)<0\right\} .
\end{aligned}
$$

Let $\left\{\ell_{t}: 0 \leq t \leq T\right\}$ be the local time at 0 of the process $X$. The event $A$ means that the path $X$ crossed zero between 0 and $T$. Hence, $A=\left\{\ell_{T}>0\right\}$. If $x=0$, then $\mathbb{P}[A]=1$. Of course, $A_{n} \subset A$ and $\mathbb{P}\left[A \backslash \cup_{n \geq} A_{n}\right]=0$. On the complementary event $\bar{A}_{n}$ of $A_{n}$, Lemma 2 asserts that the MLE $\theta_{n}$ is equal to 1 .

Let $X$ be a SBm defined as the strong solution to $(1)$ on a probability space $(\Omega, \mathcal{F}, \mathbb{P})$ for a canonical Brownian motion $W$ with a filtration $\left\{\mathcal{F}_{t}: t \geq 0\right\}$ which satisfy the usual hypotheses.

Our main theorem is the following. It proves the convergence of the MLE $\theta_{n}$ to 0 and specify its speed of convergence. In addition, this show that the MLE may be expanded as a series in terms of $1 / n^{1 / 4}$, where $\alpha_{n}$ plays a fundamental role.

Before stating our theorem, we review the notion stable convergence due to A. Renyi (see Aldous \& Eagleson (1978); Jacod \& Shiryaev (1987); Rényi (1963)).

Definition 1 (Stable convergence). Consider a sequence of random variables $Y, Y_{1}, Y_{2}, \ldots$ defined on a probability space $(\Omega, \mathcal{F}, \mathbb{P})$, and a $\sigma$-algebra $\mathcal{G} \subset \mathcal{F}$.

We say that the sequence of random variables $Y_{1}, Y_{2} \ldots$ converge $\mathcal{G}$-stably in distribution to a random variable $Y$ defined on an extension $\left(\Omega^{\prime}, \mathcal{F}^{\prime}, \mathbb{P}^{\prime}\right)$ of $(\Omega, \mathcal{F}, \mathbb{P})$, and denote

$$
Y_{n} \underset{n \rightarrow \infty}{\stackrel{\mathcal{G} \text {-stably }}{\longrightarrow}} Y
$$


when

$$
\mathbb{E}\left(Z f\left(Y_{n}\right)\right) \underset{n \rightarrow \infty}{\longrightarrow} \mathbb{E}(Z f(Y))
$$

for any bounded $\mathcal{G}$ measurable random variable $Z$, and any bounded and continuous function $f$.

Theorem 1. Assume that $\theta=0$, i.e. let $X$ be a Brownian motion on $[0, T]$ departing from $x$. Let $\mu_{k}$ for $k=1,2,3, \ldots$ be the constants given by (8) below.

(I) For $\lambda=-\mu_{1} / \mu_{2}$,

$$
\alpha_{n} \mathbf{1}_{A_{n}} \underset{n \rightarrow \infty}{\stackrel{\mathcal{F} \text {-stably }}{\longrightarrow}} \lambda \Upsilon_{T}
$$

with

$$
\Upsilon_{T}= \begin{cases}T^{1 / 4} W\left(\ell_{T}\right) / \ell_{T} & \text { if } \ell_{T}>0, \\ 0 & \text { if } \ell_{T}=0,\end{cases}
$$

where $W$ is a Brownian motion independent from $B$.

(II) Let $d_{n}^{(1)}, d_{n}^{(2)}, \ldots$ be a sequence of random variables given the recursive relation $d_{n}^{(1)}=1$ and

$$
d_{n}^{(m+1)}=-\sum_{k=1}^{m+1} \frac{L_{n}^{(k+1)}(0)}{L_{n}^{(2)}(0)} \sum_{\substack{1 \leq i_{1}, \ldots, i_{k} \leq m \\ i_{1}+\cdots+i_{k}=m+1}} d_{n}^{\left(i_{1}\right)} \cdots d_{n}^{\left(i_{k}\right)} .
$$

Let $d^{(1)}, d^{(2)}, \ldots$ be a sequence of random variables defined recursively by $d^{(1)}=1, d^{(2)}=0$ and for $m \geq 2$,

$$
\begin{gathered}
d^{(m+1)}=-\sum_{k=1}^{m} \lambda^{(k)} \sum_{\substack{1 \leq i_{1}, \ldots, i_{k} \leq m \\
i_{1}+\ldots+i_{k}=m+1}} d_{n}^{\left(i_{1}\right)} \cdots d_{n}^{\left(i_{k}\right)} \\
\text { with } \lambda^{(2 k+1)}=\frac{T^{1 / 4} \mu_{2 k+1} W\left(\ell_{T}\right)}{\mu_{2} \ell_{T}} \text { and } \lambda^{(2 k+2)}=0 .
\end{gathered}
$$

For any integer $p \geq 0$, the vector $\left(\mathbf{1}_{A_{n}} d_{n}^{(1)}, \ldots, \mathbf{1}_{A_{n}} d_{n}^{(p+1)}\right)$ converges $\mathcal{F}$-stably to $\left(d^{(1)}, \ldots, d^{(p+1)}\right)$ depending only on $\ell_{T}$ and $W\left(\ell_{T}\right)$. Besides, for any $\epsilon>0$, there exists some integer $n_{0}$ large enough and some $K$ such that

$$
\mathbb{P}\left[n^{\frac{p}{4}+\frac{1}{2}}\left|\theta_{n}-\Theta_{n}\right| \geq K ; A_{n}\right] \leq \epsilon \text { for any } n \geq n_{0},
$$

where

$$
\Theta_{n}=\frac{\alpha_{n}}{n^{1 / 4}}+d_{n}^{(2)} \frac{\alpha_{n}^{2}}{n^{1 / 2}}+\cdots+d_{n}^{(p+1)} \frac{\alpha_{n}^{p+1}}{n^{(p+1) / 4}} .
$$

In addition, $d_{n}^{(2)}$ converges in probability to 0 and $n^{1 / 4} d_{n}^{(2)}$ is bounded.

(III) It holds that $\theta_{n} \mathbf{1}_{A_{n}}$ converges in probability to 0 under the Brownian distribution and

$$
n^{1 / 4} \theta_{n} \mathbf{1}_{A_{n}} \underset{n \rightarrow \infty}{\stackrel{\mathcal{F} \text {-stably }}{\longrightarrow}} \lambda \Upsilon_{T}
$$

Remark 2. Using the scaling property of the Brownian motion and then of the local time, and adjusting the initial condition of the process, $\Upsilon_{T}$ is equal in distribution to $\Upsilon_{1}$ for any $T>0$. 


\section{Proof of the Theorem}

\subsection{Convergence of the derivatives of the log-likelihood}

The main points in the proof of Theorem 1 follows from the asymptotic behavior of the derivative $L_{n}^{(k)}(0)$ of the log-likelihood. Let $\Phi$ be the cumulative distribution function of the standard normal distribution.

Let us note that if $X$ is a SBm of parameter $\theta$ then from the scaling property, one gets easily that the process $Y$ defined by $Y_{t}=T^{-1 / 2} X_{t T}$ is also a $\mathrm{SBm}$ of parameter $\theta$ and $\ell_{t}(Y)=T^{-1 / 2} \ell_{t}$, where $\ell_{t}(Y)$ is the symmetric local time at zero of $Y$. With this remark, it is possible to apply the results from Jacod (1998) to $Y$ with $T=1$ and then to transform then back on results on $X$.

Proposition 1. Assume that $\theta=0$ in (1), i.e. let $X$ be a Brownian motion on $[0, T]$ departing from $x$, and let $\ell$ denote its symmetric local time at zero.

(a) Assume that $k=2,4, \ldots$ Denote

$$
\mu_{k}=-2 \int_{0}^{\infty}\left[1+\frac{1}{2 k-1} \exp \left(\frac{2 k(k-1) x^{2}}{(2 k-1)^{2}}\right)\right] \Phi(-x) d x .
$$

Then

$$
\frac{L_{n}^{(k)}(0)}{n^{1 / 2}} \underset{n \rightarrow \infty}{\stackrel{\text { prob. }}{\longrightarrow}} \frac{\mu_{k}}{\sqrt{T}} \ell_{T} .
$$

(b) Assume that $k=1,3, \ldots$ Then for $\mu_{k}=C\left(h^{k}\right)$, where $C$ has been defined by (20) in Appendix and $h(x, y):=\operatorname{sgn}(x+y) \exp \left(-(2 / T)(x(x+y))^{+}\right)$, there exists a Brownian motion $W^{(k)}$ independent from $B$ such that

$$
\frac{L_{n}^{(k)}(0)}{n^{1 / 4}} \underset{n \rightarrow \infty}{\stackrel{\mathcal{F} \text {-stably }}{\longrightarrow}} \frac{\mu_{k}}{T^{1 / 4}} W^{(k)}\left(\ell_{T}\right) .
$$

Remark 3. The results of J. Jacod are given for multi-dimensional statistics. For any integer $k$, the joint convergence in probability of $n^{-1 / 2}\left(L_{n}^{(2)}(0), \ldots, L_{n}^{(2 k)}(0)\right)$ holds as well as the joint $\mathcal{F}$-stable convergence of $n^{-1 / 4}\left(L_{n}^{(1)}(0), \ldots, L_{n}^{(2 k+1)}(0)\right)$. Hence, using the property of stable convergence (see Aldous \& Eagleson (1978, Theorem 1' and subsequent Remark)), this implies the joint $\mathcal{F}$-stable convergence of

$$
\begin{aligned}
& \left(n^{-1 / 4} L_{n}^{(1)}(0), n^{-1 / 2} L_{n}^{(2)}(0), \ldots, n^{-1 / 4} L_{n}^{(2 k+1)}(0), n^{-1 / 2} L_{n}^{(2 k+2)}(0)\right) \\
& \quad \stackrel{\mathcal{F} \text {-stably }}{\longrightarrow}\left(T^{-1 / 4} \mu_{1} W^{(1)}\left(\ell_{T}\right), T^{-1 / 2} \mu_{2} \ell_{T}, \ldots, T^{-1 / 4} \mu_{2 k+1} W^{(2 k+1)}\left(\ell_{T}\right), T^{-1 / 2} \mu_{2+2} \ell_{T}\right) .
\end{aligned}
$$

The correlations between the Brownian motions $W^{(k)}$ may be computed also thanks to Theorem 1.2 in Jacod (1998), but we do not use them here.

Proof. We apply Theorem 2 in the Appendix to the process $Y_{t}=T^{-1 / 2} X_{t T}$ and then we assume that $T=1$. For $T \neq 1$, the scaling property has then to be used again. Observe that

$$
h_{k}(x, y) \leq \exp \left(-(x(x+y))^{+}\right) \leq \exp \left(|y|-\left|x \wedge x^{2}\right|\right) .
$$

We then have that (17) in Appendix holds with $a=1, \hat{h}(x)=\exp \left(-\left|x \wedge x^{2}\right|\right)$ and $r=0$, then it holds for any $r>0$. In consequence, by the after mentioned theorem, 
the convergence in (18) holds for $h=h_{k}$ with $k=2,4, \ldots$ It remains to compute the constant in (19). We have

$$
\begin{aligned}
c\left(h_{k}\right)= & \iint_{\mathbb{R}^{2}} h_{k}(x, y) p(1, y) d x d y=2 \int_{0}^{\infty} d x \int_{-\infty}^{-x} p(1, y) d y \\
& +\frac{2}{\sqrt{2 \pi}} \int_{0}^{\infty} d x \int_{-x}^{\infty} \exp \left(-\frac{1}{2} y^{2}-2 k x y-2 k x^{2}\right) d y \\
= & 2 \int_{0}^{\infty} \Phi(-x) d x \\
& +\frac{2}{\sqrt{2 \pi}} \int_{0}^{\infty} d x \exp \left(2 k(k-1) x^{2}\right) \int_{-x}^{\infty} \exp \left(-\frac{1}{2}(y+2 k x)^{2}\right) d y \\
= & 2 \int_{0}^{\infty} \Phi(-x) d x+2 \int_{0}^{\infty} \exp \left(2 k(k-1) x^{2}\right) \Phi(-(2 k-1) x) d x \\
= & 2 \int_{0}^{\infty} \Phi(-x) d x+\frac{2}{2 k-1} \int_{0}^{\infty} \exp \left(\frac{2 k(k-1) x^{2}}{(2 k-1)^{2}}\right) \Phi(-x) d x .
\end{aligned}
$$

Taking into account that $\mu_{k}=-c\left(h_{k}\right)$ we conclude that (9) holds with $\mu_{k}$ given in (8).

To prove (b) we rely on Theorem 3 in the appendix. Observe then that $c\left(h_{k}\right)=0$ for odd $k$ due to the property

$$
h_{k}(-x,-y)=-h_{k}(x, y) \text { for odd } k .
$$

In view of the fact that (17) holds for all $h_{k}$ with $r=4$, we conclude the proof.

The proof of the following corollary is immediate from Proposition 1 since $A=\left\{\ell_{T}>\right.$ 0 , Remark 3 and the continuous mapping theorem.

Corollary 1. Given the conditions of Proposition 1, for $k=0,1,2, \ldots$, we have:

$$
\begin{gathered}
\frac{L_{n}^{(2 k+1)}(0)}{L_{n}^{(2)}(0)} \mathbf{1}_{A_{n}} \underset{n \rightarrow \infty}{\stackrel{\text { prob. }}{\longrightarrow}} 0, \quad \frac{L_{n}^{(2 k+2)}(0)}{L_{n}^{(2)}(0)} \mathbf{1}_{A_{n}} \frac{\text { prob. }}{n \rightarrow \infty} \frac{\mu_{2 k+2}}{\mu_{2}} \mathbf{1}_{A_{n}}, \\
n^{1 / 4} \frac{L_{n}^{(2 k+1)}(0)}{L_{n}^{(2)}(0)} \mathbf{1}_{A_{n}} \stackrel{\mathcal{F} \text {-stably }}{\underset{n \rightarrow \infty}{\longrightarrow}} T^{1 / 4} \frac{\mu_{2 k+1} W\left(\ell_{T}\right)}{\mu_{2} \ell_{T}} \mathbf{1}_{A} .
\end{gathered}
$$

In particular, for $\lambda=-\mu_{1} / \mu_{2}$

$$
-n^{1 / 4} \frac{L_{n}^{(1)}(0)}{L_{n}^{(2)}(0)} \mathbf{1}_{A_{n}} \underset{n \rightarrow \infty}{\stackrel{\mathcal{F} \text {-stably }}{\longrightarrow}} T^{1 / 4} \lambda \frac{W\left(\ell_{T}\right)}{\ell_{T}} \mathbf{1}_{A} .
$$

Hence, part (I) of Theorem 1 is immediate from this corollary.

\subsection{Asymptotic development of the MLE}

We now study the development of the MLE $\theta_{n}$ as a series in $1 / n^{1 / 4}$. This will prove Point

(II) of Theorem 1. Point (III) is an immediate consequence of Point (II).

Using the result of Remark 3, we consider the asymptotic behavior of the vector

$$
\left(n^{-1 / 4} L_{n}^{(1)}(0), n^{-1 / 2} L_{n}^{(2)}(0), \ldots, n^{-1 / 2} L_{n}^{(2 k)}(0)\right)
$$


for some $k \geq 1$. We may then consider a probability space $(\widehat{\Omega}, \widehat{\mathcal{F}}, \widehat{\mathbb{P}})$ such that this sequence is equal in distribution to a sequence converging almost surely to $\left(T^{-1 / 4} \mu_{1} W^{(1)}\left(\ell_{T}\right), T^{-1 / 2} \mu_{2} \ell_{T}, \ldots, T^{-1 / 2}\right.$ We now consider some point in this probability space such that $\ell_{T}>0$. If the starting point is 0 , then the event $\left\{\ell_{T}>0\right\}$ is of full measure.

Proposition 2. On the probability space $(\widehat{\Omega}, \widehat{\mathcal{F}}, \widehat{\mathbb{P}})$ above, the random sequences $d_{n}^{(i)}$ given by (6) are convergent and bounded in $n$. Besides, for $m=1,2,3, \ldots$,

$$
\theta_{n}=\frac{\alpha_{n}}{n^{1 / 4}}+d_{n}^{(2)} \frac{\alpha_{n}^{2}}{n^{2 / 4}}+d_{n}^{(3)} \frac{\alpha_{n}^{3}}{n^{3 / 4}}+\cdots+d_{n}^{(m)} \frac{\alpha_{n}^{m}}{n^{m / 4}}+\mathrm{O}\left(\frac{1}{n^{(m+1) / 4}}\right)
$$

almost surely in the event $\left\{\ell_{T}>0\right\}$.

Let us start by a simple lemma to get a control over the finite Taylor expansion of $L_{n}^{(1)}(\theta)$

Lemma 4. For any $\theta$ and any integer $m \geq 1$, there exists a random constant $C$ such that

$$
\left|L_{n}^{(1)}(\theta)-\sum_{k=0}^{m} L_{n}^{(k+1)}(0) \theta^{k}\right| \leq \sup _{|\xi| \leq|\theta|}\left|L_{n}^{(m+2)}(\xi)\right| \cdot|\theta|^{m+1} \leq C n^{1 / 2} \frac{|\theta|^{m+1}}{(1-|\theta|)^{m+2}} .
$$

Proof. With (5) and for $\theta \in(-1,1)$,

$$
1-|\theta| \leq \frac{q_{\theta}\left(\Delta, X_{i}, X_{i+1}\right)}{q_{0}\left(\Delta, X_{i}, X_{i+1}\right)} \leq 1+|\theta|
$$

With (3), since $L_{n}^{(k)}(0) / n^{1 / 2}$ converges in probability (either to $\mu_{k} \ell_{T}$ or to 0 depending if $k$ is even or odd), there exists a random constant $C$ such that

$$
\left|L_{n}^{(k)}(\theta)\right| \leq \frac{n^{1 / 2} C}{(1-|\theta|)^{k}}
$$

Hence

$$
\left|L_{n}^{(1)}(\theta)-\sum_{k=0}^{m} L_{n}^{(k+1)}(0) \theta^{k}\right| \leq \sup _{|\xi| \leq|\theta|}\left|L_{n}^{(m+2)}(\xi)\right| \cdot|\theta|^{m+1} .
$$

With (9) and (10), this gives (11) because $L_{n}^{(k)}(0) / n^{1 / 2}$ is bounded in $n$.

Lemma 5. For $n$ large enough, the function $L_{n}^{(1)}(\theta)$ is invertible. Besides, for $n$ large enough, the function $\left(L_{n}^{(1)}(\theta)\right)^{-1}$ is Lipschitz in $\theta$ with a constant $8 / n^{1 / 2} \mu_{2} \ell_{T}$ on the event $A_{n}$.

Proof. With (12),

$$
-L_{n}^{(2)}(\theta) \geq\left(\sum_{i=1}^{n-1} \frac{p\left(\Delta,\left|X_{i}\right|+\left|X_{i+1}\right|\right)^{2}}{q_{0}\left(\Delta, X_{i}, X_{i+1}\right)^{2}}\right) \frac{1}{(1+|\theta|)^{2}} \geq \frac{L_{n}^{(2)}(0)}{4} .
$$

Since $L_{n}^{(2)}(0)<0$ for $n$ large enough as $n^{-1 / 2} L_{n}^{(2)}(\theta)$ converges in probability to some negative random variable (see $(9)$ ), we get that $L_{n}^{(1)}(\theta)$ is one-to-one. With the formula $\partial_{\theta}\left(L_{n}^{(1)}(\theta)\right)^{-1}=1 / L_{n}^{(2)}\left(L_{n}^{(1)}(\theta)\right),\left(L_{n}^{(1)}(\theta)\right)^{-1}$ is Lipschitz in $\theta$ with constant $4 / L_{n}^{(2)}(0)$. 
The idea of the proof is then the following: We construct an estimator $\Theta_{n}$ such that for some constant $C$ and $p \geq 0$,

$$
\sup _{n \in \mathbb{N}} n^{p / 4}\left|L_{n}^{(1)}\left(\Theta_{n}\right)\right| \leq C .
$$

Since $L_{n}^{(1)}\left(\theta_{n}\right)=0$, for $n$ large enough,

$$
\begin{aligned}
\left|\Theta_{n}-\theta_{n}\right|=\left|\left(L_{n}^{(1)}\right)^{-1}\left(L_{n}^{(1)}\left(\Theta_{n}\right)\right)-\left(L_{n}^{(1)}\right)^{-1}\left(L_{n}^{(1)}\left(\theta_{n}\right)\right)\right| & \\
& \leq \frac{8 T^{1 / 2}}{n^{1 / 2} \mu_{2} \ell_{T}}\left|L_{n}^{(1)}\left(\Theta_{n}\right)\right| \leq \frac{8 C T^{1 / 2}}{n^{p / 4+1 / 2} \mu_{2} \ell_{T}} .
\end{aligned}
$$

Proof of Proposition 2. For the sake of simplicity, let us set $q=n^{1 / 4}$.

Set $\Theta_{n}=\alpha_{n} q+\beta_{n} q^{2}+\gamma_{n} q^{3}+\xi_{n} q^{4}$ for some $\beta_{n}, \gamma_{n}$ and $\xi_{n}$ to be carefully chosen. Here, we consider only the first terms in the development of $\theta_{n}$. It is easily to convince oneself that this method may be applied to any order and that the involved terms $\beta_{n}, \gamma_{n}, \xi_{n}, \ldots$ may be computed recursively and give rise to (6).

With (11) and $m=4$, there exists a constant $C$ such that

$$
\begin{aligned}
\mid L_{n}^{(1)}\left(\Theta_{n}\right)-L_{n}^{(1)}(0)-L_{n}^{(2)} & (0) \Theta_{n} \\
& \quad-L_{n}^{(3)}(0) \Theta_{n}^{2}-L_{n}^{(4)}(0) \Theta_{n}^{3}-L_{n}^{(5)}(0) \Theta_{n}^{4} \mid \leq C n^{1 / 2} \frac{\left|\Theta_{n}\right|^{5}}{\left(1-\left|\Theta_{n}\right|\right)^{6}} .
\end{aligned}
$$

Remark that $L_{n}^{(1)}(0)-L_{n}^{(2)}(0) \alpha_{n} q=0$. In order to get rid of the terms in $q^{2}$, set

$$
\beta_{n}=\frac{-L_{n}^{(3)}(0)}{L_{n}^{(2)}(0)} \alpha_{n}^{2}
$$

Since the joint distribution of $\alpha_{n}$ and $n^{1 / 4} L_{n}^{(3)}(0) / L_{n}^{(2)}(0)$ converges stably, $n^{1 / 4} \beta_{n}$ converges stably. Also, $\beta_{n}$ converges to 0 .

In order to get rid of the terms in $q^{3}$, set

$$
\gamma_{n}=-\frac{L_{n}^{(4)}(0)}{L_{n}^{(2)}(0)} \alpha_{n}^{3}-\frac{L_{n}^{(3)}(0)}{L_{n}^{(2)}(0)} \alpha_{n} \beta_{n} .
$$

From Corollary 1, $\gamma_{n}$ converges stably since $\alpha_{n}$ and $\beta_{n}$ converges stably.

In order to get rid of the terms in $q^{4}$, set

$$
\xi_{n}=-2 \frac{L_{n}^{(3)}(0)}{L_{n}^{(2)}(0)}\left(\alpha_{n} \gamma_{n}+\beta_{n}^{2}\right)-4 \frac{L_{n}^{(4)}(0)}{L_{n}^{(2)}(0)} \alpha_{n}^{2} \beta_{n} .
$$

Again, $\xi_{n}$ converges thanks to Corollary 1.

Hence

$$
L_{n}^{(1)}\left(\Theta_{n}\right)=\sum_{r=5}^{20} q^{r} B_{n}^{(r)}+R_{n}\left(\Theta_{n}\right),
$$

where $R_{n}\left(\Theta_{n}\right) \leq n^{1 / 2}\left|\Theta_{n}\right|^{5} /\left(1-\left|\Theta_{n}\right|\right)^{6}$ and $B_{n}^{(r)}$ are terms that depend linearly on $L_{n}^{(k)}(0)$ and on the power of the $\alpha_{n}, \beta_{n}, \gamma_{n}$ and $\xi_{n}$. Since the $L_{n}^{(k)}(0) / n^{1 / 2}$ are bounded, we obtain that the $n^{3 / 4} B_{n}^{(r)}$ are bounded. 
In addition, $n^{1 / 4} \Theta_{n}$ is bounded in $n$, so that $n^{3 / 4} R_{n}\left(\Theta_{n}\right)$ is bounded in $n$. With (13), this proves that for some constant $K$,

$$
\left|\Theta_{n}-\theta_{n}\right| \leq \frac{K}{n^{5 / 4}}
$$

Finally, let us note that $\beta_{n}=\alpha_{n}^{2} d_{n}^{(2)}$ with $d_{n}^{(2)}=-L_{n}^{(3)}(0) / L_{n}^{(2)}(0)$. With (1), $d_{n}^{(2)}$ converges in probability to 0 and $n^{1 / 4} d_{n}^{(2)}$ is bounded in $n$. Remark that $\gamma_{n}=\alpha_{n}^{3} d_{n}^{(3)}$ and $\xi_{n}=\alpha_{n}^{4} d_{n}^{(4)}$ where $d_{n}^{(3)}$ and $d_{n}^{(4)}$ are bounded in $n$. This result may be generalized to any order.

Proof of Theorem 1 Point (II). On the event $\left\{\ell_{T}>0\right\}$, the local time has a density (see Lemma 6 below). For each $\epsilon>0$, one may find a set $\Omega(\epsilon)$ as well as some values $0<a^{\prime}<b^{\prime}$ and $c^{\prime}$ such that $\omega \in \Omega(\epsilon)$ implies that $\ell_{T}^{x} \in\left(a^{\prime}, b^{\prime}\right)$ and $\left|W\left(\ell_{T}\right) / \ell_{T}\right| \leq c^{\prime}$ and

$$
\widehat{\mathbb{P}}\left[\Omega(\epsilon) \mid\left\{\ell_{T}>0\right\}\right] \geq 1-\epsilon / 2 .
$$

From the joint convergence of the $n^{1 / 4} L_{n}^{(2 k+1)}(0)$ to $\mu_{2 k+1} W\left(\ell_{T}\right)$ and the joint convergence of the $n^{1 / 2} L_{n}^{(2 k)}(0)$ to $\mu_{2 k} \ell_{T}$, we get for any $\epsilon>0$, there exists $0<k<a^{\prime}$ and $K>b^{\prime}$ as well as a measurable set $\Omega^{\prime}(\epsilon, n) \subset \Omega(\epsilon)$ such that

$$
\begin{aligned}
& L_{n}^{(k)}(0) \leq K \sqrt{n} \text { on } \Omega^{\prime}(\epsilon, n) \\
& L_{n}^{(2)}(0) \geq k \sqrt{n} \text { on } \Omega^{\prime}(\epsilon, n) \\
& \text { and } \forall n \geq n_{0}, \widehat{\mathbb{P}}\left[\Omega^{\prime}(n, \epsilon) \mid\left\{\ell_{T}>0\right\}\right] \geq 1-\epsilon .
\end{aligned}
$$

In the proof of Proposition 2, we constructed some estimator $\Theta_{n}$ such that for some $p \geq 0, n^{p} L_{n}^{(1)}\left(\Theta_{n}\right)$ is bounded by some constant depending the upper bounds of the $n^{1 / 2} L_{n}^{(k)}(0)$. Besides, we use the Lipschitz constant of $\left(L_{n}^{(1)}\right)^{-1}$ which depends on the lower bound of $n^{1 / 2} L_{n}^{(2)}(0)$. Thus, on $\Omega^{\prime}(\epsilon, n)$, we obtain that $\left|\theta_{n}-\Theta_{n}\right| \leq C / n^{p+1 / 2}$, where $C$ depends only on $K$ and $k$, assuming that $n \geq n_{0}$. This means that

$$
n^{p+1 / 2}\left|\theta_{n}-\Theta_{n}\right| \leq C \text {. }
$$

Thus, for any $\epsilon>0$, there exists $n_{0}$ large enough such that

$$
\forall n \geq n_{0}, \widehat{\mathbb{P}}\left[n^{p+1 / 2}\left|\theta_{n}-\Theta_{n}\right| \geq C\right] \leq \epsilon .
$$

which yields the result.

\subsection{The contrast function}

In order to study the maximum likelihood, it is also possible to consider the contrast function

$$
Z_{n}(\theta)=\frac{\exp \left(L_{n}(\theta)\right)}{\exp \left(L_{n}(0)\right)}
$$

Using the asymptotic development of $L_{n}(u)$ around 0 , we get that

$$
\log Z_{n}(\theta)=\theta L_{n}^{(1)}(0)+\frac{\theta^{2}}{2} L_{n}^{(2)}(0)+\mathrm{O}\left(\theta^{3}\right) .
$$

Thus, with the result of Proposition 1, we see that

$$
\log Z_{n}\left(\theta / n^{1 / 4}\right) \underset{n \rightarrow \infty}{\stackrel{\mathcal{F} \text {-stably }}{\longrightarrow}}-\mu_{2}\left(T^{-1 / 4} \lambda \theta W\left(\ell_{T}\right)-T^{-1 / 2} \frac{\theta^{2}}{2} \ell_{T}\right) .
$$


From this convergence we can intuitively check our result in (7), based in the theory of convergence of statistical experiments and the LAMN property in (14). The theory states (under certain stringent conditions that we do not verify) that the maximum likelihood estimator of the pre-limit experiments converges stably to the maximum likelihood estimator of the limit experiment (Ibragimov \& Has'minskii, 1981). It is direct, differentiating with respect to $\theta$ in the r.h.s. of (14), to obtain, when $\ell_{T}>0$, that the MLE in the limit experiment is $T^{1 / 4} \lambda W\left(\ell_{T}\right) / \ell_{T}$. We then obtain (7).

\section{The limit distribution}

In this section, we consider that the starting point is 0 and then that $\mathbb{P}[A]=1$. Otherwise, on the event $A$, one may use the strong Markov property at the first time the process hit zero.

As $n^{1 / 4} \theta_{n}$ and $\alpha_{n}$ converge to $\lambda \Upsilon_{T}$ with $\Upsilon_{T}=T^{1 / 4} W\left(\ell_{T}\right) / \ell_{T}$, we give the main characteristics of this random variable. As noted in Remark 2 , thanks to the scaling property of the Brownian motion, we see that $\Upsilon$ is equal in distribution to $W\left(\ell_{1}\right) / \ell_{1}$, whatever the value of $T$.

Indeed, this random variable is easy to simulate.

Lemma 6. Under $\mathbb{P}_{0}$, the distribution of $\Upsilon$ is symmetric. Besides, its density is

$$
f_{\Upsilon}(x)=\frac{\mathrm{d} F_{\Upsilon}(x)}{\mathrm{d} x}=\int_{0}^{+\infty} \mathrm{d} y \int_{0}^{1} \frac{\sqrt{y}}{2 \pi \sqrt{t^{3}}} \exp \left(\frac{-x y}{2}-\frac{y^{2}}{2 t}\right) \mathrm{d} t
$$

and it is equal in distribution to

$$
\Upsilon=\frac{G(H)}{H} \text { with } H=\frac{1}{2}\left(U+\sqrt{V+U^{2}}\right),
$$

where $G(H), U$ and $V$ are independent random variables, $G(H) \sim \mathcal{N}(0, H), U \sim \mathcal{N}(0,1)$ and $V \sim \exp (1 / 2)$.

Proof. It is well known that the local time $\ell_{1}$ at time 1 is equal in distribution to the supremum of the Brownian motion $\sup _{r \in[0,1]} B_{r}$ on $[0,1]$. It follows that

$$
F_{\ell_{1}}(y)=\mathbb{P}_{0}\left[\ell_{1}<y\right]=\mathbb{P}_{0}\left[\sup _{r \in[0,1]} B_{r}<y\right]=\mathbb{P}_{0}\left[\tau_{y}>1\right]
$$

where $\tau_{y}=\inf \left\{t>0 \mid B_{t}=y\right\}$. The density $v(t ; y)$ of $\tau_{y}$ is equal to

$$
v(t ; y)=\frac{1}{\sqrt{2 \pi t^{3}}} \exp \left(-\frac{y^{2}}{2 t}\right)
$$

so that

$$
F_{\ell_{1}}(y)=1-\int_{0}^{1} \frac{1}{\sqrt{2 \pi t^{3}}} \exp \left(-\frac{y^{2}}{2 t}\right) \mathrm{d} t
$$

and the density $f_{\ell_{1}}(y)$ of $\ell_{1}$ is then equal to

$$
f_{\ell_{1}}(y)=\int_{0}^{1} \frac{2 y}{\sqrt{2 \pi t^{3}}} \exp \left(-\frac{y^{2}}{2 t}\right) \mathrm{d} t .
$$


Thus, conditioning with respect to the value of $\ell_{1}$,

$$
F_{\Upsilon}(x)=\mathbb{P}[\Upsilon<x]=\int_{0}^{+\infty} \mathbb{P}[W(y)<x y] f(y) \mathrm{d} y
$$

and this leads to (15).

Expression (16) follows from the equality in distribution of $\ell_{1}$ and $\frac{1}{2}\left(U+\sqrt{V+U^{2}}\right)$. This expression has been used in Lépingle (1993) and Lépingle (1995) in order to simulate the reflected Brownian motion.

The variance of $\Upsilon$ is 3.16 . We see in Figure 1 that the density of $\Upsilon$ is close to that of the normal distribution, yet narrower.

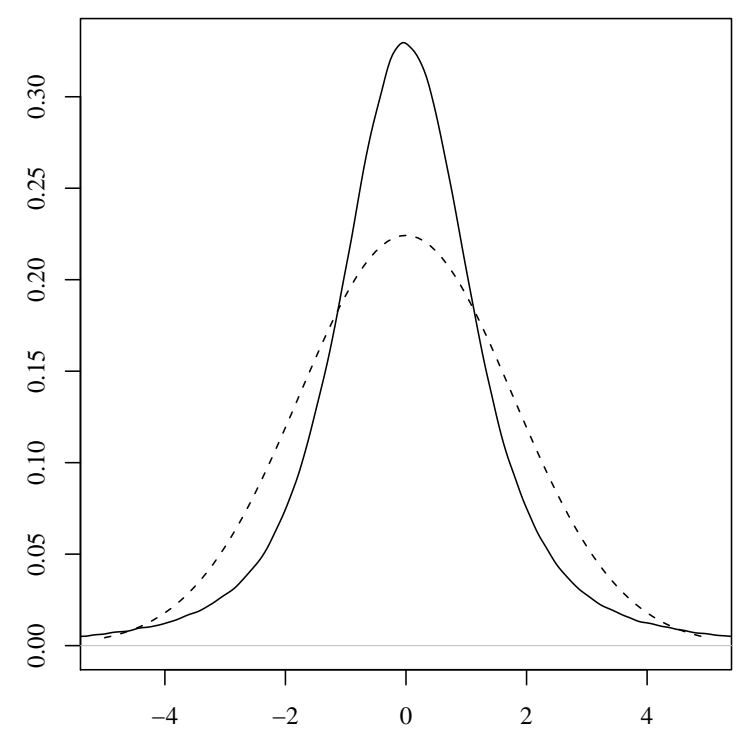

Figure 1: Density of $\Upsilon$ (solid) and density of the normal distribution with variance $\operatorname{Var}(\Upsilon)$ (dashed).

\section{$5 \quad$ Numerical tests and observations on the estimators}

In order to simplify the notations, let us set

$$
\widetilde{\alpha}_{n}=\frac{\alpha_{n}}{n^{1 / 4}}=-\frac{L_{n}^{(1)}(0)}{L_{n}^{(2)}(0)} \underset{n \rightarrow \infty}{\stackrel{\text { proba. }}{\longrightarrow}} 0 .
$$

Numerical tests are easy to perform, as all the formulae are easy to implement. For example, the SBm is easily simulated: it could be approximated by random walks (Harrison \& Shepp, 1981) or by the exact simulation algorithm proposed in Lejay \& Pichot (2012). As pointed out, the estimator $\widetilde{\alpha}_{n}$ is easily computed from the data, while the MLE may be found by a numerical optimization procedure since the density (2) of the $\mathrm{SBm}$ has a simple, analytic expression. In Section 4, we saw how to simulate random variates distributed as $\Upsilon$. 


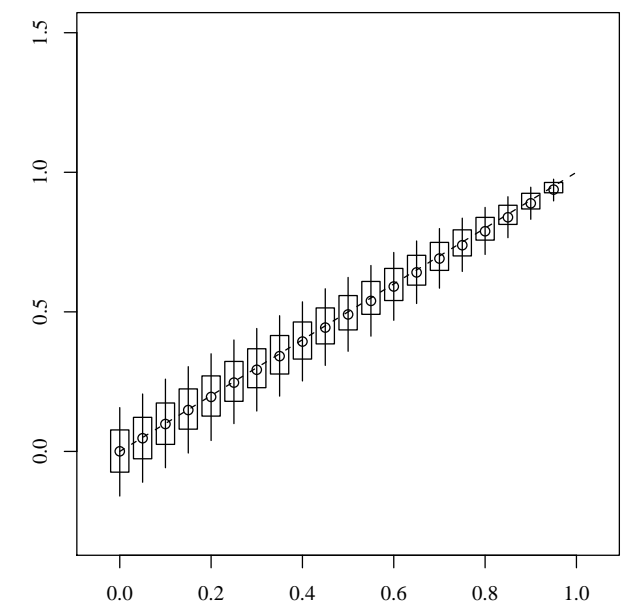

(a) $\theta_{n}$

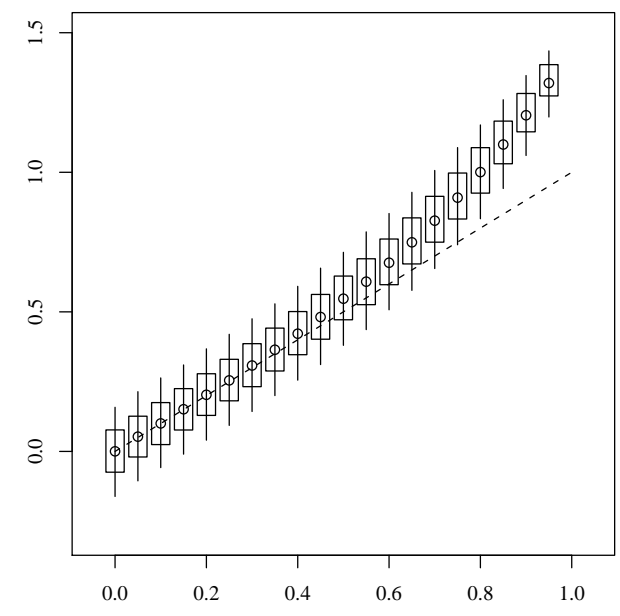

(b) $\widetilde{\alpha}_{n}$

Figure 2: Statistics of $\theta_{n}$ and $\widetilde{\alpha}_{n}$ : mean value (points), 1/4 and 3/4-quantiles (the upper and lower boundaries of the rectangles) as well as the $1 / 10$ and $9 / 10$-quantiles (the extremities of the segments) for $n=10,000$ over the 10,000 realizations of paths.

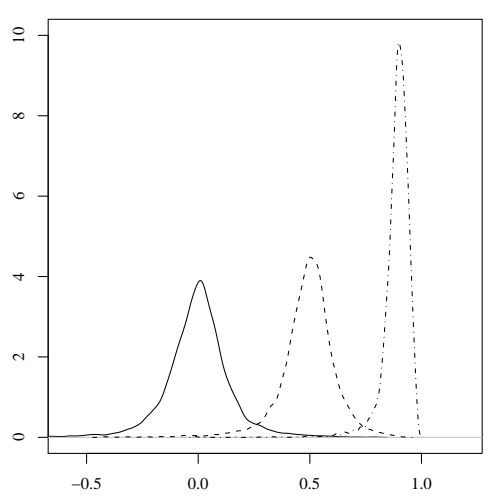

(a) $\theta_{n}$

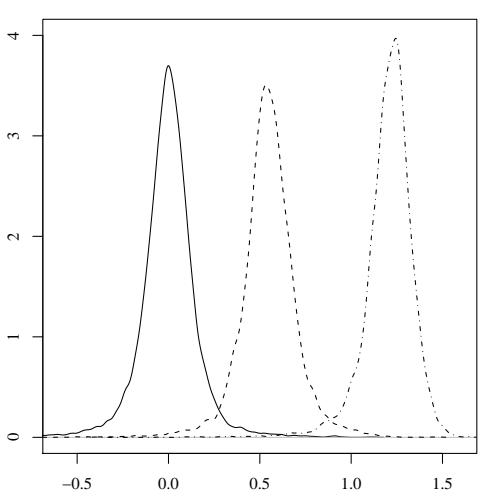

(b) $\widetilde{\alpha}_{n}$

Figure 3: Density of $\theta_{n}$ and $\widetilde{\alpha}_{n}$ for $\theta=0, \theta=5 / 10$ and $\theta=9 / 10$ and $n=10,000$ sample points.

\subsection{Numerical behavior of the estimators for the SBM with $\theta \neq 0$}

Of course, a natural question which arise is to know the behavior of the MLE $\theta_{n}$ and of $\widetilde{\alpha}_{n}$ when the observed points $\left\{X_{i}\right\}_{i=0, \ldots, n}$ arise from a SBm of parameter $\theta \neq 0$.

As noted in the introduction of this Section, numerical tests could easily be performed for any value of $\theta$, so that one may for example construct some hypothesis test with thresholds given numerical estimation of quantiles. This could indeed give better values than the one obtained by simply using an asymptotic theorem.

In Figure 2, we plot for values of the skewness parameter $\theta$ in the set $\left\{0, \frac{5}{100}, \ldots, \frac{95}{100}\right\}$ the mean values of $\theta_{n}$ and $\widetilde{\alpha}_{n}$ over 10,000 paths with $n=10,000$ samples points. We also plot its $1 / 10,1 / 4,3 / 4$ and $9 / 10$-quantiles.

In Figure 3, we plot the density of $\theta_{n}$ and $\widetilde{\alpha}_{n}$ for $\theta \in\left\{0, \frac{5}{10}, \frac{9}{10}\right\}$ for $n=10,000$ sample points and constructed from $N=10,000$ paths. We see that the density of $\theta_{n}$ is skewed for $\theta \neq 0$, as one could expect, and that the variance of $\theta_{n}$ seems to decrease as $\theta$ increase to one. This is not the case with $\widetilde{\alpha}_{n}$. 


\begin{tabular}{|c|rrrrrrrrrr|}
\hline$n$ & 100 & 250 & 500 & 1,000 & 2,500 & 5,000 & 10,000 & 50,000 & 100,000 & 250,000 \\
\hline 1/10-q. & -0.59 & -0.76 & -1.01 & -1.06 & -1.15 & -1.20 & -1.17 & -1.34 & -0.94 & -1.48 \\
\hline 9/10-q. & 0.65 & 0.89 & 0.91 & 0.98 & 1.11 & 1.13 & 1.35 & 1.34 & 1.23 & 1.33 \\
\hline Sdev & 1.20 & 1.94 & 2.89 & 4.38 & 6.38 & 9.95 & 13.00 & 31.95 & 30.80 & 80.47 \\
\hline
\end{tabular}

Table 1: Quantiles and standard deviation for of $n^{3 / 4}\left(\theta_{n}-\widetilde{\alpha}_{n}\right)$ over $N=10,000$ paths.

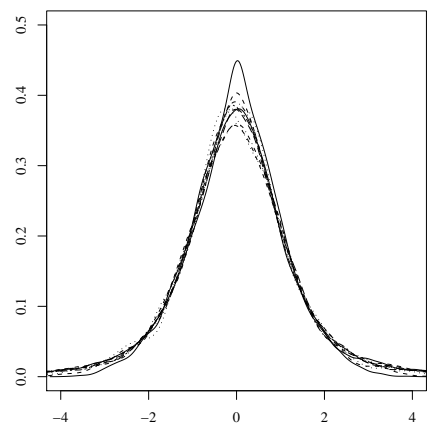

(a) Densities

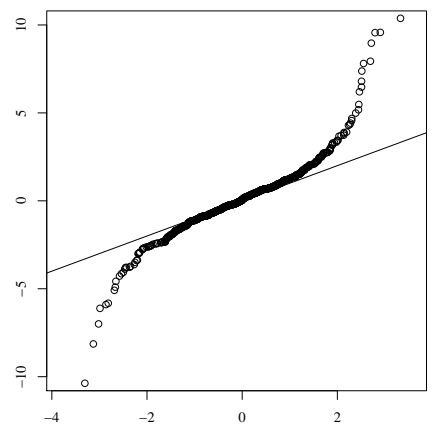

(b) Q-Q for $\alpha_{100}$ vs. $\alpha_{250000}$

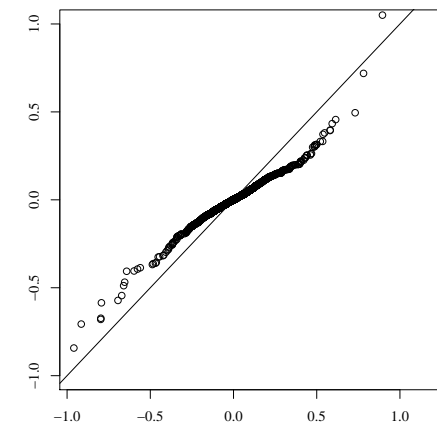

(c) Q-Q for $\alpha_{250000}$ vs. $c \Upsilon$

Figure 4: Densities of $\alpha_{n}=n^{1 / 4} \widetilde{\alpha}_{n}$ for the values of $n$ give in Table 1 and QuantileQuantile plot of $\alpha_{n}$ for $n=250,000$ against $n=100$ and $\alpha_{n}$ against $c \Upsilon$ with $c$ such that $\operatorname{Var}\left(\alpha_{n}\right)=\operatorname{Var}(c \Upsilon)$. The straight lines are defined by $y=x$ and show the concordance.

We see that the average value of $\theta_{n}$ is close $\theta$ and that the MLE seems to be a consistent estimator of $\theta$. On the other hand, the mean value of $\widetilde{\alpha}_{n}$ is close to $\theta$ only for small values of $\theta$ (say $\theta<5 / 10$ ) and overestimates $\theta$ for larger values.

\subsection{Study of the estimator for small values of $n$ and $\theta=0$}

Theorem 1 asserts that $\theta_{n}-\widetilde{\alpha}_{n}$ is of order $\mathrm{O}\left(1 / n^{3 / 4}\right)$. In Table 1 , we give the $1 / 10$ and 9/10-quantiles of $n^{3 / 4}\left(\theta_{n}-\widetilde{\alpha}_{n}\right)$. for small values of $n$. However, the standard deviation increases with $n$.

We also see in Figure 4(a) that the density of $\alpha_{n}=n^{1 / 4} \widetilde{\alpha}_{n}$ remains stable with values of $n$, even for small values of $n$. Figure 4(b) presents Quantile-Quantile plot for $\alpha_{100}$ against $\alpha_{250000}$, which confirms the closeness of these two distributions. In Figure 4(c), we see that the distribution of $\alpha_{250000}$ is also close to the one of $\Upsilon=c W\left(\ell_{1}\right) / \ell_{1}$ with $c$ chosen to get equality of the variances.

\section{An example of application: diffusion of species}

As endowed in the introduction, the $\mathrm{SBm}$ is a fundamental tool when one has to model a permeable barrier. In addition, it appears when one writes down the processes generated by diffusion equations with discontinuous coefficients in a one dimensional media: this issue is presented in the survey article Lejay (2006) with references to the articles where the SBm arises and covering various fields, such as ecology, finance, astrophysics, geophysics, ...

We present here a possible application to ecology of our hypothesis test, which can be surely applied to other fields. 


\subsection{Has a boundary between two habitats an effect?}

Diffusions are commonly used in ecology to explain the spread of a specie, at the level of individual cells (See for example the book Berg (1993)) or the level of an animal in a wild environment.

Several authors have proposed the use of biased diffusions to model the behavior of a specie at the boundary between two habitats, such as Cantrell \& Cosner (1999); Ovaskainen \& Cornell (2003), when the species diffuse with different species at speed in each habitat.

Now, consider a situation where the dispersion of a specie in two different habitats is well modelled by a diffusion process, and that the measurement of the diffusion coefficient give the same value. Does it means that the boundary has no effect on the displacement of the individuals?

Let us apply this in a one-dimensional world, where one habitat is $[0,+\infty)$ and the other is $(-\infty, 0]$. We assume that we may track the position of an individual, whose displacement in each of the habitat is given by $x+\sigma B_{t}$.

Then, we may apply our hypothesis test to determine whether or not the position shall be modelled by

$$
\left(\mathrm{H}_{0}\right) \quad X_{t}=x+\sigma B_{t}
$$

or by

$$
\left(\mathrm{H}_{1}\right) \quad X_{t}=x+\sigma B_{t}+\theta \ell_{t}^{0}(X) .
$$

Under Hypothesis $\left(\mathrm{H}_{0}\right)$, the boundary has no effect and is not seen. Under Hypothesis $\left(\mathrm{H}_{1}\right)$, the individual is more likely to go in one of the two habitat, depending on the sign of $\theta$.

\subsection{What is the underlying operator?}

Now, let us consider that we have a measurement of the diffusion coefficients that gives two different values $a_{+}$on $\mathbb{R}_{+}$and $a_{-}$on $\mathbb{R}_{-}$.

One may then wonder which differential operator shall be used to model the diffusive behavior. For $a=a_{+} \mathbf{1}_{[0,+\infty)}+a_{-} \mathbf{1}_{(-\infty, 0)}$, is it

$$
L=\frac{1}{2} \nabla(a \nabla \cdot) \text { or } A=\frac{1}{2} a \triangle \quad ?
$$

On $(0,+\infty)$ and $(-\infty, 0)$, there is no difference between these two operators, which means that the local dynamic of the particle/individual is not affected by the choice of $L$ and $A$. However, the difference arises at 0 : the process $X$ generated by $L$ is solution to

$$
X_{t}=x+\int_{0}^{t} \sqrt{a\left(X_{s}\right)} \mathrm{d} B_{s}+\frac{a_{+}-a_{-}}{a_{+}+a_{-}} \ell_{t}^{0}(X)
$$

while the process $Y$ generated by $A$ is solution to

$$
Y_{t}=x+\int_{0}^{t} \sqrt{a\left(X_{s}\right)} \mathrm{d} B_{s},
$$

for a Brownian motion $B$ (See for example Lejay (2006); Lejay \& Martinez (2006)). From the analytic point of view: the $\operatorname{domain} \operatorname{Dom}(A)$ of $A$ contains the functions of class $\mathcal{C}^{2}(\mathbb{R})$ which are bounded with bounded, first and second order derivatives. The domain 
$\operatorname{Dom}(L)$ of $L$ contains functions of class $\mathcal{C}^{2}(\mathbb{R} \backslash\{0\})$ with bounded first and second order derivatives which are furthermore continuous at 0 , and such that $a_{+} \nabla f(0+)=a_{-} \nabla f(0-)$. This condition is called the flux condition. In many physical situations, it is assumed that the flux $a \nabla u$ is continuous and this is why divergence-form operators of type $L$ arise.

Remark 4 . Both $L$ and $A$ can be embedded in a single class of operators of type $\frac{\rho}{2} \nabla(a \nabla \cdot)$. If $\rho$ and $a$ are constant on $(0,+\infty)$ and $(-\infty, 0)$, then we may use the following characterization: let us consider

$$
C=\frac{1}{2} \nabla(a \nabla \cdot) \text { with } a=a_{+} \mathbf{1}_{[0,+\infty)}+a_{-} \mathbf{1}_{(-\infty, 0)}
$$

and

$$
\operatorname{Dom}(C)=\left\{\begin{array}{l|l}
f \in \mathcal{C}^{2}(\mathbb{R} \backslash\{0\}) & \begin{array}{l}
f, f^{\prime}, f^{\prime \prime} \text { are bounded on } \mathbb{R} \backslash\{0\} \\
f(0-)=f(0+) \\
(1+\lambda) f^{\prime}(0+)=(1-\lambda) f^{\prime}(0-), \lambda \in(-1,1)
\end{array}
\end{array}\right\} .
$$

This class of operators is then specified by three parameters, $a_{+}>0, a_{-}>0$ and $\lambda \epsilon$ $(-1,1)$. The operator $A$ corresponds to $\lambda=0$, while $L$ corresponds to $\lambda=\left(a_{+}-a_{-}\right) /\left(a_{+}+\right.$ $\left.a_{-}\right)$.

For $\Phi(x)=\int_{0}^{x} \mathrm{~d} x / \sqrt{a(x)}, \widehat{X}=\Phi(X)$ is solution to the SDE (Lejay, 2006; Lejay \& Martinez, 2006)

$$
\widehat{X}_{t}=\Phi(x)+B_{t}+\frac{\sqrt{a_{+}}-\sqrt{a_{-}}}{\sqrt{a_{+}}+\sqrt{a_{-}}} \ell_{t}^{0}(\widehat{X})
$$

while $\widehat{Y}=\Phi(Y)$ is solution to the SDE

$$
\widehat{Y}_{t}=\Phi(x)+B_{t}+\frac{\sqrt{a_{-}}-\sqrt{a_{+}}}{\sqrt{a_{+}}+\sqrt{a_{-}}} \ell_{t}^{0}(\widehat{Y})
$$

We then see that both $\widehat{X}$ and $\widehat{Y}$ are Skew Brownian motions, but the coefficients in front of their local time have opposite signs.

Even if we have not studied the asymptotic behavior of the MLE for the SBm with skewness parameter different from 0 , numerical experiments back the following hypotheses test:

1. Given an observed $X$, estimate the diffusion coefficient for the process on each side of 0 .

2. Apply the function $\Phi$ to the observed process.

3. Compute the MLE $\theta_{n}$ of the Skewness parameter. If $a_{+}>a_{-}$(resp. $a_{+}<a_{-}$) and $\theta_{n}>0$ then decided that the infinitesimal generator of $X$ is $L$ (resp. A). Otherwise, decide that it is $A$ (resp. $L$ ).

\section{Conclusion}

In this article, we have studied the behavior of the maximum likelihood for the Skew Brownian motion when the parameter to estimate is 0 . 
In particular, we have shown that the rate of convergence of the estimator $\theta_{n}$ is $n^{1 / 4}$ and not $n^{1 / 2}$ as in the classical case. This should not be surprising: indeed, away from 0 , the Skew Brownian motion behaves like a Brownian motion, and only its dynamic close to 0 allows one to see the difference between a Skew Brownian motion with a parameter $\theta \neq 0$ and a Brownian motion. It is also not surprising that the local time enters in the limit distribution.

The case $\theta \neq 0$ remains open. One needs to prove results similar to the one of Jacod (1998), when the Brownian motion is replaced by the Skew Brownian motion (its distribution with respect to the Wiener measure is singular). Of course, one cannot expect the limit law to be symmetric. Yet, it is pretty easy to simulate the Skew Brownian motion and to estimate the maximum likelihood, so that numerical studies are easy to perform.

\section{Appendix}

In this Appendix we provide the theorems given in Jacod (1998) used for the proofs of the main results in Section 2. We slightly change the notation and present the results in the particular cases that are relevant to us in the present work.

Denote by $X=\left\{X_{t}: 0 \leq t \leq 1\right\}$ a Brownian motion on a probability space $(\Omega, \mathcal{F}, \mathbb{P})$. Introduce a Borel function $h: \mathbb{R}^{2} \rightarrow \mathbb{R}$ such that there exist $a \in \mathbb{R}$ and $h: \mathbb{R} \rightarrow \mathbb{R}$ such that

$$
h(x, y) \leq e^{a|y|} \hat{h}(x) \quad \text { and } \quad \int|x|^{r}|\hat{h}(x)| d x<\infty .
$$

Theorem 2 (Jacod (1998, Theorem 1.1, p. 508)). Consider $h$ as above, satisfying (17) with $r=0$. Then

$$
\frac{1}{n^{1 / 2}} \sum_{i=0}^{n-1} h\left(\sqrt{n} X_{i / n}, \sqrt{n}\left(X_{(i+1) / n}-X_{i / n}\right)\right) \underset{n \rightarrow \infty}{\stackrel{\text { prob. }}{\longrightarrow}} c(h) \ell_{1},
$$

where

$$
c(h)=\iint_{\mathbb{R}^{2}} h(x, y) p(1, y) d x d y
$$

and $\left(\ell_{t}\right)_{t \geq 0}$ denotes the local time of $X$ at level zero.

Remark 5. It must be noticed that the convergence in (18), as stated in Jacod (1998), is stronger, in the sense that both terms in (18) are processes (i.e. depend on $t$ ) and the convergence is locally uniformly in time, in probability. Recall that a sequence $\left(Z^{n}\right)_{n \geq 1}$ of processes is said to converges locally uniformly in time, in probability, to a limiting

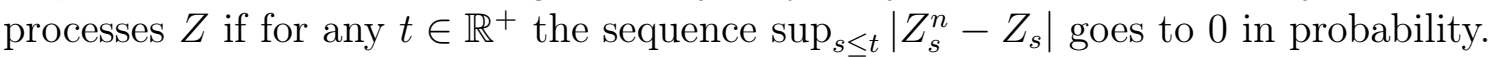

Let us assume now that $h$ is such that $c(h)=0$. Then set

$$
C(h)=c\left(h^{2}\right)+2 \int_{\mathbb{R}} \int_{\mathbb{R}} p(1, y) h(x, y) H(x+y) \mathrm{d} y \mathrm{~d} x
$$

with

$$
H(x)=\sum_{k=0}^{+\infty} \int_{\mathbb{R}} p(k, y) \int_{\mathbb{R}} p(1, z) h(x+y, z) \mathrm{d} z \mathrm{~d} y .
$$


Theorem 3 (Jacod (1998, Theorem 1.2, p. 511)). Consider $h$ as above, satisfying (17) with some $r>3$, and assume that $c(h)=0$ (see (19)). Then

$$
\frac{1}{n^{1 / 4}} \sum_{i=0}^{n-1} h\left(\sqrt{n} X_{i / n}, \sqrt{n}\left(X_{(i+1) / n}-X_{i / n}\right)\right) \underset{n \rightarrow \infty}{\stackrel{\mathcal{F} \text {-stable in dist. }}{\longrightarrow}} \sqrt{C(h)} W\left(\ell_{1}\right),
$$

where $W=\left\{W_{t}: t \geq 0\right\}$ is a Brownian motion independent of $X$, and $\left(\ell_{t}\right)_{t \geq 0}$ is the local time of $X$ at level zero. The constant $C(h)$ is given by (20).

Remark 6. As in the previous remark, the theorem stated in Jacod (1998) is stronger, now in the sense that both terms in (21) are processes, and the processes converge stably in distribution in the Skorokhod space of càdlàg functions.

\section{References}

Aldous, D. J. \& Eagleson, G. K. (1978). On mixing and stability of limit theorems. Ann. Probability 6.2, 325-331.

Bardou, O. \& Martinez, M. (2010). Statistical estimation for reflected skew processes. Stat. Inference Stoch. Process. 13.3, 231-248. DOI: 10.1007/s11203-010-9047-6.

Berg, H. (1993). Random walks in Biology. Princeton University Press.

Cantrell, R. \& Cosner, C. (1999). Diffusion models for population dynamics incorporating individual behavior at boundaries: Applications to refuge design. Theoretical Population Biology 55.2, 189-207.

Florens-Zmirou, D. (1993). On estimating the diffusion coefficient from discrete observations. J. Appl. Probab. 30.4, 790-804.

Fujihara, E., Kawamura, Y. \& Yano, Y. (2007). Functional limit theorems for occupation times of Lamperti's stochastic processes in discrete time. J. Math. Kyoto Univ. 47.2, 429-440.

Harrison, J. M. \& Shepp, L. A. (1981). On skew Brownian motion. Ann. Probab. 9.2, 309-313.

Höpfner, R. \& Löcherbach, E. (2003). Limit theorems for null recurrent Markov processes. Mem. Amer. Math. Soc. 161.768.

Ibragimov, I. \& Has'minskii, R. (1981). Statistical Estimation Asymptotic Theory. Springer. Jacod, J. \& Shiryaev, A. N. (1987). Limit theorems for stochastic processes. SpringerVerlag.

Jacod, J. (1998). Rates of convergence to the local time of a diffusion. Ann. Inst. H. Poincaré Probab. Statist. 34.4, 505-544. DOI: 10.1016/S0246-0203(98)80026-5.

Jacod, J. (2006). Parametric inference for discretely observed non-ergodic diffusions. Bernoulli 12.3, 383-401. DOI: 10.3150/bj/1151525127.

Kutoyants, Y. (1984). Parameter Estimation for Stochastic Processes. Heldermann.

Lamperti, J. (1958). An occupation time theorem for a class of stochastic processes. Trans. Amer. Math. Soc. 88, 380-387.

Le Cam, L. \& Yang, G. L. (2000). Asymptotics in statistics. 2nd ed. Springer Series in Statistics. Springer-Verlag.

Le Gall, J.-F. (1984). One-dimensional stochastic differential equations involving the local times of the unknown process. In: Stochastic analysis and applications, Swansea, 1983. Vol. 1095. Lecture Notes in Math. Springer, 51-82.

Lejay, A. (2006). On the constructions of the skew Brownian motion. Probab. Surv. 3, 413-466. DOI: $10.1214 / 154957807000000013$. 
Lejay, A. \& Martinez, M. (2006). A scheme for simulating one-dimensional diffusion processes with discontinuous coefficients. Ann. Appl. Probab. 16.1, 107-139. DOI: 10 . 1214/105051605000000656.

Lejay, A. \& Pichot, G. (2012). Simulating diffusion processes in discontinuous media: a numerical scheme with constant time steps. J. Comput. Phys. 231.21, 7299-7314. DOI: 10.1016/j.jcp.2012.07.011.

Lépingle, D. (1995). Euler scheme for reflected stochastic differential equations. Math. Comput. Simulation 38.1-3: Probabilités numériques (Paris, 1992), 119-126. DOI: 10. 1016/0378-4754 (93)E0074-F.

Lépingle, D. (1993). Un schéma d'Euler pour équations différentielles stochastiques réfléchies. C. R. Acad. Sci. Paris Sér. I Math. 316.6, 601-605.

Lipster, R. \& Shiryaev, A. (2001). Statistics of random processes. II. Applications. Springer.

Martinez, M. (2004). Interprétations probabilistes d'opérateurs sous forme divergence et analyse de méthodes numériques associées. Ph.D. thesis. Université de Provence / INRIA Sophia-Antipolis.

Ovaskainen, O. \& Cornell, S. J. (2003). Biased movement at a boundary and conditional occupancy times for diffusion processes. J. Appl. Probab. 40.3, 557-580.

Ramirez, J. M., Thomann, E. A., Waymire, E. C., Haggerty, R. \& Wood, B. (2006). A generalized Taylor-Aris formula and skew diffusion. Multiscale Model. Simul. 5.3, 786801. DOI: $10.1137 / 050642770$.

Rényi, A. (1963). On stable sequences of events. Sankhyā Ser. A 25, 293-302.

Walsh, J. (1978). A diffusion with discontinuous local time. In: Temps locaux. Vol. 52-53. Astérisques. Société Mathématique de France, 37-45.

Zhang, M. (2000). Calculation of diffusive shock acceleration of charged particles by skew Brownian motion. The Astrophysical Journal 541, 428-435.

- Antoine Lejay

Université de Lorraine, IECN, UMR 7502, Vandœuvre-lès-Nancy, F-54500, France; CNRS, IECL, UMR 7502, Vandœuvre-lès-Nancy, F-54500, France;

Inria, Villers-lès-Nancy, F-54600, France;

email: 〈Antoine.Lejay@univ-lorraine.fr $\rangle$.

- Ernesto Mordecki

Centro de Matemática, Facultad de Ciencias, Universidad de la República. Iguá 4225, 11400, Montevideo, Uruguay;

email: 〈mordecki@cmat.edu.uy .

- Soledad Torres

CIMFAV, Facultad de Ingeniería; Universidad de Valparaíso; Casilla 123-V; Chile; email: 〈soledad.torres@uv.cl〉. 